\title{
DIFFERENTIAL EQUATION FOR ASYMMETRIC VOIGT PROFILE
}

\author{
R. Ciuryeo, J. Domyslawska and R.S. Trawiński \\ Institute of Physics, Nicholas Copernicus University \\ Grudziądzka 5, 87-100 Toruń, Poland \\ (Received January 8, 1993; in final form March 4, 1993)
}

The second-order differential equation for the asymmetric Voigt profile is presented, leading to a powerful and accurate method of determining the line shape parameters.

PACS numbers: $32.70 . \mathrm{Jz}, 33.70 . \mathrm{Jg}, 34.20 . \mathrm{Cf}$

\section{Introduction}

Spectral line shapes at low pressures of perturbing gas are commonly interpreted in terms of the Voigt profile $V(a ; x)$ which is a convolution of Lorentzian and Gaussian profiles [1]

where

$$
V(a ; x)=\frac{a}{\pi} \int_{-\infty}^{+\infty} \frac{\exp \left[-(x-y)^{2}\right]}{y^{2}+a^{2}} \mathrm{~d} y
$$

$$
\begin{aligned}
& a=\left(\sqrt{\ln 2} \Gamma_{\mathrm{L}}\right) / \Gamma_{\mathrm{D}}, \\
& x=\left[2\left(\omega-\omega_{0}-\Delta\right) \sqrt{\ln 2}\right] / \Gamma_{\mathrm{D}} .
\end{aligned}
$$

Here $\Gamma_{\mathrm{L}}$ and $\Gamma_{\mathrm{D}}$ are the Lorentzian and Doppler widths, respectively, and $\Delta$ is the pressure shift of the maximum of the Lorentzian component of the Voigt profile with respect to the unperturbed frequency $\omega_{0}$ of the line. The Lorentzian width $\Gamma_{\mathrm{L}}$ and shift $\Delta$ are linear functions of the perturbing gas density. Contrary to that, the Doppler width $\Gamma_{\mathrm{D}}$ does not depend on density but is specified by the temperature $T$ of a gas and the mass $M$ of the radiating atom

$$
\Gamma_{\mathrm{D}}=\frac{2 \omega_{0}}{c}\left(\frac{2 k T \ln 2}{M}\right)^{1 / 2}
$$

The corresponding Doppler profile $I_{\mathrm{D}}(\omega)$ is then identical to the Gaussian distribution

$$
I_{\mathrm{D}}(\omega)=\frac{2 \sqrt{\ln 2}}{\sqrt{\pi} \Gamma_{\mathrm{D}}} \exp \left\{-4 \ln 2\left[\left(\omega-\omega_{0}\right) / \Gamma_{\mathrm{D}}\right]^{2}\right\} .
$$


Since the Voigt profile has been taken as the basis for detailed analysis of spectral line shapes, first of all in applications to plasma diagnostics and astrophysics, many algorithms for fast and accurate tabulating of $V(a ; x)$ have been developed in recent years [2-4]. Moreover, Andersen [5] and Squire [6] have derived a differential equation which is satisfied by the Voigt function $V(a ; x)$. It should be emphasized, however, that the Voigt profile is valid if two conditions are fulfilled. Firstly, the radiation emitted during the time of collision between radiating and perturbing atoms is neglected. This corresponds strictly to the impact approximation of the pressure broadening theory. Secondly, the Doppler broadening arising from the thermal motion is uncorrelated with the pressure broadening. If these two conditions are not met then as was shown in Refs. [7-12], deviations from the Voigt profile are expected which may lead to an asymmetry of the resultant line shape in the near wing and core region.

The present paper deals with the so-called collision time asymmetry arising from the breakdown of the impact approximation. As was shown in Refs. [10-12], the first-order correction to the usual impact (i.e. Lorentzian) line shape has a dispersion form so that the shape of pressure broadened line can be written as

$$
I(\omega)=\frac{\Gamma_{\mathrm{L}}}{2 \pi} \frac{1+\beta\left(\omega-\omega_{0}-\Delta\right)}{\left(\omega-\omega_{0}-\Delta\right)^{2}+\left(\Gamma_{\mathrm{L}} / 2\right)^{2}},
$$

where the asymmetry parameter $\beta$ is independent of pressure and is proportional to a collision time (cf. [13-16]). This parameter contains information on the interatomic interaction potentials and the collision dynamics. It should be noted that the asymmetry parameter $\beta$ is expected to show the same sign as the Lorentzian shift $\Delta$.

The resultant line shape $F(a, b ; x)$ including the ordinary Doppler broadening may be written in the form

$$
F(a, b ; x)=\frac{a}{\pi} \int_{-\infty}^{+\infty} \frac{\exp \left[-(x-y)^{2}\right]}{y^{2}+a^{2}}(1+b y) \mathrm{d} y,
$$

where

$$
b=\left(\beta \Gamma_{\mathrm{D}}\right) /(2 \sqrt{\ln 2}) .
$$

Hereafter the above profile will be referred to as asymmetric Voigt profile (AVP). The main purpose of this work is to derive a differential equation enabling fast tabulating of the AVP which would be more effective than the direct calculating of the integral (7).

\section{Differential equation}

Let us consider the function $H(a ; x)$ given by

$$
H(a ; x)=\frac{a}{\pi} \int_{-\infty}^{+\infty} \frac{\exp \left[-(x-y)^{2}\right]}{y^{2}+a^{2}} f(y) \mathrm{d} y
$$

where $a$ is the real, positive, non-zero parameter, and $f(y)$ is the $C_{\infty}$ class function. One can show that this function satisfies the following differential equation:

$$
\frac{\partial^{2}}{\partial x^{2}} H+4 x \frac{\partial}{\partial x} H+\left(4 a^{2}+4 x^{2}+2\right) H=\frac{4 a}{\pi} \zeta(x),
$$


where $\zeta(x)$ is the functional series

$$
\zeta(x)=\sum_{k=0}^{\infty} \frac{1}{k !} f^{(k)}(x) C_{k},
$$

which is assumed to be uniformly convergent. The coefficients $C_{k}$ in (11) are defined by

$$
C_{k}=\int_{-\infty}^{+\infty} t^{k} \mathrm{e}^{-t^{2}} \mathrm{~d} t
$$

It is easy to test that for $k$ being natural number $C_{k}$ has the following properties:

$$
\begin{aligned}
& C_{0}=\sqrt{\pi}, \\
& C_{2 k+1}=0, \\
& C_{2 k}=\frac{(2 k-1) ! !}{2^{k}} C_{0} .
\end{aligned}
$$

Equation (10) is defined on the same range as the series $\zeta(x)$ and may be calculated easily if the $f(x)$ in (11) is a polynominal, sine or cosine function.

\section{The asymmetric Voigt profile (AVP)}

Let us consider a function $W_{n}(a ; x)$ given by a formula

$$
W_{n}(a ; x)=\frac{a}{\pi} \int_{-\infty}^{+\infty} \frac{\exp \left[-(x-y)^{2}\right]}{y^{2}+a^{2}}\left(\frac{y}{a}\right)^{n} \mathrm{~d} y .
$$

As results from Eqs. (10) and (11) this function satisfies the following differential equation:

$$
\frac{\partial^{2}}{\partial x^{2}} W_{n}+4 x \frac{\partial}{\partial x} W_{n}+\left(4 a^{2}+4 x^{2}+2\right) W_{n}=\frac{4 a^{1-n}}{\pi} \sum_{k=0}^{n}\left(\begin{array}{c}
n \\
k
\end{array}\right) x^{n-k} C_{k} .
$$
follows:

The asymmetric Voigt profile $F(a, b ; x)$ given by Eq. (7) may be written as

$$
F(a, b ; x)=W_{0}(a ; x)+b a W_{1}(a ; x) .
$$

Keeping in mind that the appropriate constants satisfy Eqs. (13)-(15) and using Eq. (17), we get the differential equation for the AVP function

$$
\frac{\partial^{2}}{\partial x^{2}} F+4 x \frac{\partial}{\partial x} F+\left(4 a^{2}+4 x^{2}+2\right) F=\frac{4 a}{\sqrt{\pi}}(1+b x)
$$

which for $b=0$ turns into the equation for the usual Voigt profile given by Andersen [5]. In order to solve the above differential equation it is necessary to state the appropriate initial conditions for the function $F(a, b ; x)$ and its first derivative. In our case it is convenient to introduce the following initial conditions:

$$
\begin{aligned}
& F(a, b ; 0)=\frac{a}{\pi} \int_{-\infty}^{+\infty} \frac{\exp \left(-y^{2}\right)}{y^{2}+a^{2}} \mathrm{~d} y \\
& {\left[\frac{\partial}{\partial x} F(a, b ; x)\right]_{x=0}=\frac{2 a b}{\pi} \int_{-\infty}^{+\infty} \frac{\exp \left(-y^{2}\right)}{y^{2}+a^{2}} y^{2} \mathrm{~d} y .}
\end{aligned}
$$


The above formulas can be easily evaluated using Eq. (7). Using. the Fourier transforms of the Gaussian and asymmetrical Lorentzian profiles, the $F(a, b ; x)$ function may be rewritten in the following way:

$$
F(a, b ; x)=\frac{1}{\sqrt{\pi}} \int_{0}^{+\infty} \exp \left(a t-t^{2} / 4\right)[\cos (x t)+a b \sin (x t)] \mathrm{d} t .
$$

The conditions (20) and (21) can be rewritten as

$$
\begin{aligned}
& F(a, b ; 0)=\exp \left(a^{2}\right)[1-\operatorname{erf}(a)], \\
& {\left[\frac{\partial}{\partial x} F(a, b ; x)\right]_{x=0}=2 a b\left[\frac{1}{\sqrt{\pi}}-a F(a, b ; 0)\right],}
\end{aligned}
$$

where

$$
\operatorname{erf}(a)=\frac{2}{\sqrt{\pi}} \int_{0}^{a} \mathrm{e}^{-t^{2}} \mathrm{~d} y
$$

is the error function. As it is seen in Eqs. (23) and (24), in order to determine the initial conditions we have to calculate only one integral defining the erf (a) value. The system of three differential equations which are satisfied by the Voigt profile has been given by Andersen [5]. In the Appendix we present the system of equations which is satisfied by the $W_{1}(a ; x)$ function.

Let us note that for tabulating the $F(a, b ; x)$ function, it is more effective to solve the differential equation (19), because in the next step of calculations we use the values formerly calculated. On the other hand, if we need the value of the $F(a, b ; x)$ function only for one $x$ value, it is more convenient to calculate the appropriate integral. We have found that for $a=4.9$ and $b=0.01$ the computation time for calculating the AVP profile on the basis of Eq. (19) in the range between $x=0$ and $x=20$ with 0.05 step was six times shorter than that in case of calculations based on the integral formulas, Eq. (7). Both calculations were performed with the similar accuracy. The ratio of the computation speeds in these two types of calculation depends on the required accuracy and number of $x$-points. If the tabulating step is 0.01 then the speed ratio appears to be more than 15 . We made a comparison of the results obtained using Eq. (19) and substituting $b=0$, with the values reported by Bakshi and Kearney [17]. No differences were displayed. Let us note that for large $a$-values the ability of exact computing of the initial conditions from the Eq. (23) is limited by the numerical accuracy of the used computer. For PC computers with math coprocessor, for which the real numbers are represented on no more than 10 bytes, the upper limiting $a$-value is about 5 . In the case of $a>5$ we should compute the initial conditions from the integral formula of Eq. (20). In the calculations all the integrals were evaluated using 6-point Newton-Cotes quadrature, while the differential equation was solved using the Runge-Kutta method.

The authors wish to thank Prof. J. Szudy and Prof. A. Bielski for their valuable help in preparation of this manuscript.

This work was supported by a grant No. $575 / 2 / 91$ from the Committee for Scientific Research. 


\section{Appendix}

Let us consider the complex function $\mathcal{V}(z)$ given by a formula

$$
\mathcal{V}(z)=\frac{1}{\sqrt{\pi}} \int_{0}^{\infty} \exp \left(-z t-t^{2} / 4\right) \mathrm{d} t
$$

where

$$
z=a+\mathrm{i} x .
$$

This function satisfies the following differential equation:

$$
z \mathcal{V}-\frac{1}{2} \frac{\partial}{\partial z} \mathcal{V}=\frac{1}{\sqrt{\pi}}
$$

Let us note that the functions $W_{0}$ and $W_{1}$ are

$$
\begin{aligned}
& W_{0}=\operatorname{Re} \mathcal{V}, \\
& W_{1}=-\operatorname{Im} \mathcal{V} .
\end{aligned}
$$

The Voigt profile may be written as follows:

$$
V(a ; x)=W_{0}(a ; x) .
$$

For this function, using Eqs. (A3) and (A4), we obtain the equations derived by Andersen [5]:

$$
\begin{aligned}
& \frac{\partial^{2}}{\partial x^{2}} V+4 x \frac{\partial}{\partial x} V+\left(4 a^{2}+4 x^{2}+2\right) V=\frac{4 a}{\sqrt{\pi}}, \\
& \frac{\partial^{2}}{\partial a^{2}} V-4 a \frac{\partial}{\partial a} V+\left(4 a^{2}+4 x^{2}-2\right) V=\frac{4 a}{\sqrt{\pi}} \\
& \frac{\partial^{2}}{\partial x^{2}} V+\frac{\partial^{2}}{\partial a^{2}} V=0 .
\end{aligned}
$$

Using Eqs. (A3) and (A5) we can show that the $W_{1}(a ; x)$ function satisfies the similar system of equations

$$
\begin{aligned}
& \frac{\partial^{2}}{\partial x^{2}} W_{1}+4 x \frac{\partial}{\partial x} W_{1}+\left(4 a^{2}+4 x^{2}+2\right) W_{1}=\frac{4 x}{\sqrt{\pi}} \\
& \frac{\partial^{2}}{\partial a^{2}} W_{1}-4 a \frac{\partial}{\partial a} W_{1}+\left(4 a^{2}+4 x^{2}-2\right) W_{1}=\frac{4 x}{\sqrt{\pi}} \\
& \frac{\partial^{2}}{\partial x^{2}} W_{1}+\frac{\partial^{2}}{\partial a^{2}} W_{1}=0 .
\end{aligned}
$$

\section{References}

[1] A.C.G. Mitchell, M.W. Zemansky, Resonance Radiation and Excited Atoms, Cambridge University Press, Cambridge 1971.

[2] J.T. Twitty, P.L. Rarig, R.E. Thomson, J. Quant. Spectrosc. Radiat. Transf. 24, $529(1980)$.

[3] A.K. Hui, B.H. Armstrong, A.A. Wray, J. Quant. Spectrosc. Radiat. Transf. 19, 509 (1978). 
[4] A. Klim, J. Quant. Spectrosc. Radiat. Transf. 26, 537 (1981).

[5] T. Andersen, J. Quant. Spectrosc. Radiat. Transf. 19, 169 (1978).

[6] W. Squire, J. Quant. Spectrosc. Radiat. Transf. 23, 531 (1980).

[7] P.R. Berman, J. Quant. Spectrosc. Radiat. Transf. 12, 1313 (1972).

[8] E. Bielicz, E. Czuchaj, J. Fiutak, Acta Phys. Pol. A 41, 327 (1972).

[9] J. Ward, J. Cooper, E.W. Smith, J. Quant. Spectrosc. Radiat. Transf. 14, 555 (1974).

[10] P.W. Anderson, J.D. Talman, Conference on Broadening of Spectral Lines, University of Pittsburg, unpublished (1955).

[11] J. Szudy, W.E. Baylis, J. Quant. Spectrosc. Radiat. Transf. 15, 641 (1975); 17, 681 (1977).

[12] G. Peach, Adv. Phys. 30, 367 (1981); J. Phys. B, At. Mol. Phys. 17, 2599 (1984).

[13] M. Harris, E.L. Lewis, D. McHugh, I. Shannon, J. Phys, B, At. Mol. Phys. 17, L661 (1984); 19, 3207 (1986).

[14] I. Shannon, M. Harris, D.R. McHugh, E.L. Lewis J. Phys. B, At. Mol. Phys. 19, 1409 (1986).

[15] R.E. Walkup, A. Spielfiedel, D.E. Pritchard, Phys. Rev. Lett. 45, 986 (1980).

[16] R.E. Walkup, B. Stewart. D.E. Pritchard, Phys. Rev. A 29, 169 (1984).

[17] V. Bakshi, R.J. Kearney, J. Quant. Spectrosc. Radiat. Transfer. 42, 111 (1989). 